Bull. Korean Math. Soc. 49 (2012), No. 2, pp. 271-284

http://dx.doi.org/10.4134/BKMS.2012.49.2.271

\title{
LINEAR WEINGARTEN SPACELIKE HYPERSURFACES IN LOCALLY SYMMETRIC LORENTZ SPACE
}

\author{
DAN YANG
}

\begin{abstract}
Let $M$ be a linear Weingarten spacelike hypersurface in a locally symmetric Lorentz space with $R=a H+b$, where $R$ and $H$ are the normalized scalar curvature and the mean curvature, respectively. In this paper, we give some conditions for the complete hypersurface $M$ to be totally umbilical.
\end{abstract}

\section{Introduction}

Let $N_{1}^{n+1}$ be an $(n+1)$-dimensional pseudo-Riemannian manifold of index 1 , which is called Lorentz space. When the Lorentz space $N_{1}^{n+1}$ is of constant curvature $c$, we call it Lorentz space form, denoted by $N_{1}^{n+1}(c)$. A hypersurface $M$ of a Lorentz space $N_{1}^{n+1}$ is said to be spacelike if the induced metric on $M$ from that of the Lorentz space is positive definite.

It is well know that spacelike hypersurfaces in a Lorentz space form have been investigated by many differential geometers from both the physical and the mathematical points of view. Goddard [9] conjectured that a complete spacelike hypersurface in de Sitter space $N_{1}^{n+1}(1)$ with constant mean curvature $H$ must be totally umbilical. Akutagawa [2] and Ramanathan [17] proved independently that the conjecture is true if $H^{2} \leq 1$ when $n=2$ and $n^{2} H^{2} \leq$ $4(n-1)$ when $n \geq 3$. In [14], Montiel proved that Goddard's conjecture is true provided that $M^{n}$ is compact. Montiel [15] proved that complete spacelike hypersurface $M^{n}$ with $H^{2}=4(n-1) / n^{2}$ is isometric to a hyperbolic cylinder if $M^{n}$ has at least two ends. Another natural Goddard-like problem is to study hypersurfaces of Lorentze space with constant scalar curvature. An interesting result of Cheng and Ishikawa [8] states that the totally umbilical round spheres are the only compact spacelike hypersurfaces in de Sitter space $N_{1}^{n+1}(1)$ with constant normalized scalar curvature $R<1$. Some other authors, such as Brasil-Colares-Palmas [4], Camargo-Chaves-Sousa Jr [5], Caminha [6] and Li [10] have also worked on related problems.

Received October 21, 2010; Revised February 13, 2011.

2010 Mathematics Subject Classification. 53C42.

Key words and phrases. linear Weingarten, spacelike hypersurface, locally symmetric. 
When $M^{n}$ is a complete spacelike hypersurface in de Sitter space $N_{1}^{n+1}(1)$ with $R=k H$, Cheng [7] proved that if the sectional curvature is non-negative and $H$ can obtain its maximum on $M^{n}$, then $M^{n}$ is totally umbilical. Shu [18] obtained a characteristic theorem concerning such hypersurfaces in terms of the mean curvature.

All of the above results were obtained under the assumption that the ambient manifolds possess very nice symmetry properties. Many researchers have recently begun to study ambient manifolds which do not have symmetry in general, such as locally symmetric Lorentz space, see [3], [13], [12], [19], [20] and [21]. First of all, we recall that, for constants $c_{1}$ and $c_{2}$, Jin Ok Baek et al. [3] introduced the class of $(n+1)$-dimensional Lorentz spaces $N_{1}^{n+1}$ of index 1 which satisfy the following two conditions (here and in the sequel, $\bar{K}$ denotes the sectional curvature on $N_{1}^{n+1}$ ):

(1) for any spacelike vector $\mu$ and timelike vector $\nu$,

$$
\bar{K}(\mu, \nu)=-c_{1} / n ;
$$

(2) for any spacelike vector $\mu$ and timelike vector $\nu$,

$$
\bar{K}(\mu, \nu) \geq c_{2} .
$$

There are several examples of Lorentz spaces satisfying (1.1) and (1.2), for instance,

Example 1.1. The Lorentz space form $N_{1}^{n+1}(c)$, where $-c_{1} / n=c_{2}=c$.

Example 1.2. Semi-Riemannian product manifold $H_{1}^{k}\left(-c_{1} / n\right) \times N^{n+1-k}\left(c_{2}\right)$, $c_{1}>0$, and $R_{1}^{k} \times S^{n+1-k}(1)$. In particular, $R_{1}^{1} \times S^{n}(1)$ is so-called Einstein Static Universe.

Example 1.3. Robertson-Walker spacetime $N(c, f)=I \times_{f} N^{3}(c)$, where $I$ denotes an open interval of $R_{1}^{1}$ and $f>0$ a smooth function defined on the interval $I, N^{3}(c)$ a 3-dimensional Riemannian manifold of constant curvature c.

We denote by $\bar{K}_{C D}$ the components of the Ricci tensor of $N_{1}^{n+1}$ satisfying (1.1) and (1.2), then the scalar curvature $\bar{R}$ of $N_{1}^{n+1}$ is given by

$$
\bar{R}=\sum_{A=1}^{n+1} \varepsilon_{A} \bar{K}_{A A}=-2 \sum_{i=1}^{n} \bar{K}_{n+1 i i n+1}+\sum_{i, j=1}^{n} \bar{K}_{i j j i}=2 c_{1}+\sum_{i, j=1}^{n} \bar{K}_{i j j i} .
$$

It is well known $\bar{R}$ is constant when Lorentz space $N_{1}^{n+1}$ is locally symmetric, so $\sum_{i, j=1}^{n} \bar{K}_{i j j i}$ is constant.

A hypersurface in Lorentz space $N_{1}^{n+1}$ is called linear Weingarten hypersurface if $c R+d H+e=0$, where $c, d, e$ are constants such that $c^{2}+d^{2} \neq 0$. When the constant $d$ vanishes, linear Weingarten hypersurfaces reduce to hypersurfaces with constant scalar curvature; When the constant $c$ vanishes, linear Weingarten hypersurfaces reduce to hypersurfaces with constant mean curvature; When the constant $e$ vanishes, linear Weingarten hypersurfaces reduce to 
hypersurfaces with $R=k H$. In [11], Li-Suh-Wei studied compact linear Weingarten hypersurfaces with $R=a H+b$ in unit sphere $S^{n+1}(1)$. In this paper, we will consider spacelike linear Weingarten hypersurfaces with $R=a H+b$ in locally symmetric Lorentz space $N_{1}^{n+1}$. Our results as following generalizes some known ones.

Theorem 1.4. Let $N_{1}^{n+1}$ be a locally symmetric Lorentz space satisfying (1.1) and (1.2), $M^{n}$ is a complete spacelike linear Weingarten hypersurface immersed in $N_{1}^{n+1}$ with $R=a H+b$ satisfying $a \neq 0, b<c_{2}$. Suppose that the maximum of $H$ can be attained on $M^{n}$. If the sectional curvature of $M^{n}$ is not less than $-c_{1} / n-c_{2}$, then $M^{n}$ is totally umbilical or an isometric hypersurface with two distinct principle curvature, one of which is simple.

In particular, when $N_{1}^{n+1}$ is de Sitter space $N_{1}^{n+1}(c)\left(c=-c_{1} / n=c_{2}>0\right)$, from Theorem 1.4 we have the following corollary by means of the congruence Theorem of Abe-Koike-Yamaguchi [1].

Corollary 1.5. Let $M^{n}$ be a complete spacelike linear Weingarten hypersurface immersed in $N_{1}^{n+1}(c)$ with $R=a H+b$ satisfying $a \neq 0, b<c$. Suppose that the maximum of $H$ can be attained on $M^{n}$. If the sectional curvature of $M^{n}$ is non-negative, then $M^{n}$ is totally umbilical or $H^{1}\left(c_{1}\right) \times S^{n-1}\left(c_{2}\right)$, where $1 / c_{1}+1 / c_{2}=1 / c$.

Theorem 1.6. Let $N_{1}^{n+1}$ be a locally symmetric Lorentz space satisfying (1.1) and (1.2). $M^{n}$ is a complete spacelike linear Weingarten hypersurface immersed in $N_{1}^{n+1}$ with $R=a H+b$ satisfying $b<c_{2}$. If $S \leq 2 \sqrt{n-1}\left(2 c_{2}+c_{1} / n\right)$, then either

(1) $M^{n}$ is totally umbilical, or

(2) $\sup S=2 \sqrt{n-1}\left(2 c_{2}+c_{1} / n\right)$. If $\sup S$ is attained at some point in $M^{n}$, then $M^{n}$ is isometric to an isometric hypersurface with two distinct principle curvature, one of which is simple.

\section{Preliminaries}

Let $M^{n}$ be an $n$-dimensional spacelike hypersurface immersed in the Lorentz space $N_{1}^{n+1}$. We choose a local field of pseudo-Riemannian orthonormal frames $\left\{e_{1}, \ldots, e_{n+1}\right\}$ in $N_{1}^{n+1}$ such that, restricted to $M^{n}, e_{1}, \ldots, e_{n}$ are tangent to $M^{n}$, and the vector $e_{n+1}$ is normal to $M^{n}$. Let $\left\{\omega_{1}, \ldots, \omega_{n+1}\right\}$ be the dual frame field. In this paper, we make the following convention on the range of indices:

$$
1 \leq A, B, C \leq n+1 ; \quad 1 \leq i, j, k \leq n .
$$

Then the structure equations of $N_{1}^{n+1}$ are given by

$$
\begin{aligned}
& d \omega_{A}=-\sum_{B} \varepsilon_{B} \omega_{A B} \wedge \omega_{B}, \quad \omega_{A B}+\omega_{B A}=0, \\
& d \omega_{A B}=-\sum_{C} \varepsilon_{C} \omega_{A C} \wedge \omega_{C B}-\frac{1}{2} \sum_{C, D} \varepsilon_{C} \varepsilon_{D} \bar{K}_{A B C D} \omega_{C} \wedge \omega_{D},
\end{aligned}
$$


where $\varepsilon_{i}=1, \varepsilon_{n+1}=-1$ and $\bar{K}_{A B C D}$ denotes the components of the Riemannian curvature tensor of $N_{1}^{n+1}$. Then

$$
\bar{K}_{C D}=\sum_{B} \varepsilon_{B} \bar{K}_{B C D B}, \quad \bar{K}=\sum_{A} \varepsilon_{A} \bar{K}_{A A} .
$$

Next we define the covariant derivative of $K_{A B C D}$ by

$$
\begin{array}{r}
\sum_{E} \varepsilon_{E} \bar{K}_{A B C D ; E} \omega_{E}=d \bar{K}_{A B C D}-\sum_{E} \varepsilon_{E}\left(\bar{K}_{E B C D} \omega_{E A}+\bar{K}_{A E C D} \omega_{E B}\right. \\
\left.+\bar{K}_{A B E D} \omega_{E C}+\bar{K}_{A B C E} \omega_{E D}\right) .
\end{array}
$$

We restrict these forms to the spacelike hypersurface $M^{n}$ in $N_{1}^{n+1}$ and have $\omega_{n+1}=0$. The induced metric $d s^{2}$ of $M$ is written as $d s^{2}=\sum_{i} \omega_{i}^{2}$. We may put

$$
\omega_{n+1 i}=\sum_{j} h_{i j} \omega_{j}, \quad h_{i j}=h_{j i} .
$$

The quadratic form $B=\sum_{i, j} h_{i j} \omega_{i} \otimes \omega_{j} \otimes e_{n+1}$ is the second fundamental form of $M^{n}$. We denote $L=\left(h_{i j}\right)_{n \times n}$ and $S=\sum h_{i j}^{2}$. The mean curvature vector $\xi$ of $M^{n}$ is defined by

$$
\xi=\frac{1}{n} \sum_{i} h_{i i} e_{n+1} .
$$

The length of the mean curvature vector is called the mean curvature of $M^{n}$, denote by $H$. When $\xi \neq 0$, we choose $e_{n+1}$ to assure $H=\frac{1}{n} \sum_{i} h_{i i}^{n+1}>0$.

We can obtain the structure equations of $M^{n}$

$$
\begin{aligned}
& d \omega_{i}=-\sum_{j} \omega_{i j} \wedge \omega_{j}, \quad \omega_{i j}+\omega_{j i}=0, \\
& d \omega_{i j}=-\sum_{k} \omega_{i k} \wedge \omega_{k j}-\frac{1}{2} \sum_{k, l} R_{i j k l} \omega_{k} \wedge \omega_{l},
\end{aligned}
$$

and the Gauss equation

$$
R_{i j k l}=\bar{K}_{i j k l}-\left(h_{i l} h_{j k}-h_{i k} h_{j l}\right),
$$

where $\left\{R_{i j k l}\right\}$ is the component of the curvature tensor of $M^{n}$. Let $R_{i j}$ and $R$ denote the components of the Ricci curvature and the normalized scalar curvature of $M^{n}$ respectively. From (2.2) we have

$$
\begin{gathered}
R_{i k}=\sum_{j} \bar{K}_{j i k j}-n H h_{i k}+\sum_{j} h_{i j} h_{j k}, \\
n(n-1) R=\sum_{i, j} \bar{K}_{j i i j}-n^{2} H^{2}+S .
\end{gathered}
$$

Let $h_{i j k}$ denote the covariant derivative of $h_{i j}$ so that

$$
\sum_{k} h_{i j k} \omega_{k}=d h_{i j}-\sum_{k} h_{k j} \omega_{k i}-\sum_{k} h_{i k} \omega_{k j}
$$


Then by exterior differentiation of (2.1), we obtain the Codazzi equation

$$
h_{i j k}=h_{i k j}+\bar{K}_{n+1 i j k} .
$$

Next, we define the second covariant derivative of $h_{i j}$ by

$$
\sum_{l} h_{i j k l} \omega_{l}=d h_{i j k}-\sum_{m} h_{m j k} \omega_{m i}-\sum_{m} h_{i m k} \omega_{m j}-\sum_{m} h_{i j m} \omega_{m k} .
$$

By exterior differentiation of (2.5), we can get the following Ricci identity

$$
h_{i j k l}-h_{i j l k}=-\sum_{m}\left(h_{m j} R_{m i k l}+h_{i m} R_{m j k l}\right) .
$$

Restricting the covariant derivative $\bar{K}_{A B C D ; E}$ of $\bar{K}_{A B C D}$ on $M^{n}$, then $\bar{K}_{n+1 i j k ; l}$ is given by

$$
\bar{K}_{(n+1) i j k ; l}=\bar{K}_{(n+1) i j k l}+\bar{K}_{(n+1) i(n+1) k} h_{j l}+\bar{K}_{(n+1) i j(n+1)} h_{k l}+\bar{K}_{m i j k} h_{m l},
$$

where $\bar{K}_{(n+1) i j k l}$ denotes the covariant derivative of $\bar{K}_{(n+1) i j k}$ as a tensor on $M^{n}$.

The Laplacian of $h_{i j}$ is defined by $\triangle h_{i j}=\sum_{k} h_{i j k k}$. From (2.5) and (2.6) we obtain

$$
\triangle h_{i j}=n H_{i j}+\sum_{i, j, k}\left(\bar{K}_{n+1 k i k j}+\bar{K}_{n+1 i j k k}\right)-\sum_{i, j, k, m}\left(h_{m k} R_{m i j k}+h_{i m} R_{m k j k}\right) .
$$

Since $\frac{1}{2} \Delta S=\sum_{i, j, k}\left(h_{i j k}\right)^{2}+\sum_{i, j} h_{i j} \Delta h_{i j}$, then it follows from (2.7) and (2.8) that

$$
\begin{aligned}
& \frac{1}{2} \triangle S \\
= & \sum_{i, j, k} h_{i j k}^{2}+\sum_{i, j, k} n h_{i j} H_{i j}+\sum_{i, j} h_{i j}\left(\bar{K}_{n+1 i j k ; k}+\bar{K}_{n+1 k i k ; j}\right) \\
& -\left(S \sum_{k} \bar{K}_{n+1 k n+1 k}+n H \sum_{i, j} h_{i j} \bar{K}_{n+1 i j n+1}\right) \\
& -\sum_{i, j, k, m}\left(h_{i j} h_{m k} \bar{K}_{m i j k}+h_{i j} h_{m j} \bar{K}_{m k i k}+h_{i j} h_{m k} R_{m i j k}+h_{i j} h_{i m} R_{m k j k}\right) .
\end{aligned}
$$

Let $T=\sum_{i, j} T_{i j} \omega_{i} \omega_{j}$ be a symmetric tensor on $M^{n}$ defined by

$$
T_{i j}=n H \delta_{i j}-h_{i j}
$$

We introduce an operator $\square$ associated to $T$ acting on $f \in C^{2}\left(M^{n}\right)$ by

$$
\square f=\sum_{i, j} T_{i j} f_{i j}=\sum_{i, j}\left(n H \delta_{i j}-h_{i j}\right) f_{i j} .
$$


Setting $f=n H$ in (2.10) and from (2.4) we obtain

$$
\begin{aligned}
\square(n H) & =\sum_{i, j}\left(n H \delta_{i j}-h_{i j}\right)(n H)_{i j} \\
& =\sum_{i}(n H)(n H)_{i i}-\sum_{i, j} n h_{i j} H_{i j} \\
& =\frac{1}{2} \Delta(n H)^{2}-\sum_{i}\left(n H_{i}\right)^{2}-\sum_{i, j} n h_{i j} H_{i j} \\
& =\frac{1}{2} \Delta S-\frac{1}{2} n(n-1) \Delta R-n^{2}|\nabla H|^{2}-\sum_{i, j} n h_{i j} H_{i j} .
\end{aligned}
$$

We introduce another operator

$$
L=\square+\frac{n-1}{2} a \Delta .
$$

Then it follows from $R=a H+b$ and (2.11) that

$$
\begin{aligned}
L(n H) & =\square(n H)+\frac{n-1}{2} a \Delta(n H) \\
& =\square(n H)+\frac{1}{2} n(n-1) \Delta R \\
& =\frac{1}{2} \Delta S-n^{2}|\nabla H|^{2}-\sum_{i, j} n h_{i j} H_{i j} .
\end{aligned}
$$

Substituting (2.9) into (2.12) we have

$$
\begin{aligned}
& L(n H) \\
= & \sum_{i, j, k} h_{i j k}^{2}-n^{2}|\nabla H|^{2}+\sum_{i, j, k} h_{i j}\left(\bar{K}_{n+1 i j k ; k}+\bar{K}_{n+1 k i k ; j}\right) \\
& -\left(S \sum_{k} \bar{K}_{n+1 k n+1 k}+n H \sum_{i, j} h_{i j} \bar{K}_{n+1 i j n+1}\right) \\
& -\sum_{i, j, k, m}\left(h_{i j} h_{m k} \bar{K}_{m i j k}+h_{i j} h_{m j} \bar{K}_{m k i k}+h_{i j} h_{m k} R_{m i j k}+h_{i j} h_{i m} R_{m k j k}\right) .
\end{aligned}
$$

Lemma $2.1([16])$. Let $\mu_{i}(1 \leq i \leq n)$ be real numbers such that $\sum_{i} \mu_{i}=0$ and $\sum_{i} \mu_{i}^{2}=\beta^{2}$, where $\beta=$ constant $\geq 0$. Then

$$
-\frac{n-2}{\sqrt{n(n-1)}} \beta^{3} \leq \sum_{i} \mu_{i}^{3} \leq \frac{n-2}{\sqrt{n(n-1)}} \beta^{3}
$$

and the equality holds if and only if at least $(n-1)$ of the $\mu_{i}$ are equal.

Proposition 2.2. Let $M^{n}$ be an n-dimensional spacelike linear Weingarten hypersurface immersed in a locally symmetric Lorentz space $L^{n+1}$ satisfying (1.1) and (1.2) with $R=a H+b$. If $a \neq 0, b<c_{2}$, then $L$ is elliptic. 
Proof. If $H=0$, we have $R=b<c_{2}$. It follows from (2.4) that $S=n(n-$ $1) b-\sum_{i j} \bar{K}_{i j i j} \leq n(n-1)\left(b-c_{2}\right)<0$. This is impossible. Therefore we have $H>0$. It follows from $(2.4)$ and $R=a H+b$ that

$$
S=n^{2} H^{2}+n(n-1)(a H+b)-\sum_{i, j} \bar{K}_{j i i j}
$$

then

$$
a=\frac{1}{n(n-1) H}\left(S-n^{2} H^{2}-n(n-1) b+\sum_{i, j} \bar{K}_{j i i j}\right)
$$

We choose a local frame of orthonormal vector fields $\left\{e_{i}\right\}$ such that $h_{i j}=\lambda_{i} \delta_{i j}$. For any $i$, from (2.16) we have

$$
\begin{aligned}
& n H-\lambda_{i}+\frac{n-1}{2} a \\
(2.17)= & n H-\lambda_{i}+\frac{1}{2 n H}\left(S-n^{2} H^{2}-n(n-1) b+\sum_{i, j} \bar{K}_{j i i j}\right) \\
= & \left(\frac{1}{2}(n H)^{2}-n H \lambda_{i}+\frac{1}{2} S+\frac{1}{2} \sum_{i, j} \bar{K}_{j i i j}-\frac{1}{2} n(n-1) b\right)(n H)^{-1} .
\end{aligned}
$$

Since $\bar{K}_{j i i j} \geq c_{2}$, we have

$$
\begin{aligned}
& n H-\lambda_{i}+\frac{n-1}{2} a \\
\geq & \left\{\frac{1}{2}\left(\sum_{j} \lambda_{j}\right)^{2}-\lambda_{i} \sum_{j} \lambda_{j}+\frac{1}{2} \sum_{j} \lambda_{j}^{2}+\frac{1}{2} n(n-1)\left(c_{2}-b\right)\right\}(n H)^{-1} \\
= & \left\{\sum_{j} \lambda_{j}^{2}+\frac{1}{2} \sum_{l \neq j} \lambda_{l} \lambda_{j}-\lambda_{i} \sum_{j} \lambda_{j}+\frac{1}{2} n(n-1)\left(c_{2}-b\right)\right\}(n H)^{-1} \\
= & \left\{\sum_{i \neq j} \lambda_{j}^{2}+\frac{1}{2} \sum_{l \neq j, l, j \neq i} \lambda_{l} \lambda_{j}+\frac{1}{2} n(n-1)\left(c_{2}-b\right)\right\}(n H)^{-1} \\
= & \frac{1}{2}\left\{\sum_{i \neq j} \lambda_{j}^{2}+\left(\sum_{j \neq i} \lambda_{j}\right)^{2}+n(n-1)\left(c_{2}-b\right)\right\}(n H)^{-1} .
\end{aligned}
$$

It follows from $b<c_{2}$ that

$$
n H-\lambda_{i}+\frac{n-1}{2} a>0 .
$$

Thus $L$ is an elliptic operator. 
Proposition 2.3. Let $M^{n}$ be an n-dimensional spacelike linear Weingarten hypersurface immersed in a locally symmetric Lorentz space $L^{n+1}$ satisfying (1.1) and (1.2) with $R=a H+b$. If $(n-1) a^{2}+4 n\left(c_{2}-b\right) \geq 0$, then we have

$$
\sum_{i, j, k} h_{i j k}^{2} \geq n^{2}|\nabla H|^{2} \text {. }
$$

Moreover, suppose that the equality holds on $M^{n}$ in (2.19), then $H$ is constant.

Proof. From (2.4) and $R=a H+b$, we have

$$
S=n^{2} H^{2}+n(n-1)(a H+b)-\sum_{i, j} \bar{K}_{j i i j} .
$$

Since $K_{A B C D ; E}=0$, taking the covariant derivative of (2.20), we have

$$
2 \sum_{i, j} h_{i j} h_{i j k}=S_{k}=\left(2 n^{2} H+n(n-1) a\right) H_{k}
$$

for every $k$. Hence, by Cauchy-Schwartz's inequality, we have

$$
\sum_{i, j} h_{i j}^{2} \sum_{i, j, k} h_{i j k}^{2} \geq\left(n^{2} H+\frac{1}{2} n(n-1) a\right)^{2}|\nabla H|^{2},
$$

that is

$$
S \sum_{i, j, k} h_{i j k}^{2} \geq\left(n^{2} H+\frac{1}{2} n(n-1) a\right)^{2}|\nabla H|^{2} .
$$

On the other hand, it follows from (2.20) that

$$
\begin{aligned}
& \left(n^{2} H+\frac{1}{2} n(n-1) a\right)^{2}-n^{2} S \\
= & n^{2}\left(n^{2} H^{2}+n(n-1) H a-S\right)+\frac{1}{4} n^{2}(n-1)^{2} a^{2} \\
= & n^{2} \sum_{i, j} K_{j i i j}-n^{3}(n-1) b+\frac{1}{4} n^{2}(n-1)^{2} a^{2} \\
\geq & \frac{1}{4} n^{2}(n-1)\left((n-1) a^{2}+4 n\left(c_{2}-b\right)\right) .
\end{aligned}
$$

Since $(n-1) a^{2}+4 n\left(c_{2}-b\right) \geq 0$, we have

$$
\left(n^{2} H+\frac{1}{2} n(n-1) a\right)^{2} \geq n^{2} S
$$

It follows from (2.23) and (2.25) that

$$
S \sum_{i, j, k} h_{i j k}^{2} \geq\left(n^{2} H+\frac{1}{2} n(n-1) a\right)^{2}|\nabla H|^{2} \geq n^{2} S|\nabla H|^{2} .
$$

Hence either $S=0$ and $\sum_{i, j, k} h_{i j k}^{2}=n^{2}|\nabla H|^{2}$ or $\sum_{i, j, k} h_{i j k}^{2} \geq n^{2}|\nabla H|^{2}$. 
We suppose $\sum_{i, j, k} h_{i j k}^{2}=n^{2}|\nabla H|^{2}$ on $M^{n}$. Then equalities in (2.22), (2.23), (2.24), (2.25) and (2.26) hold.

If $(n-1) a^{2}+4 n\left(c_{2}-b\right)>0$, then $\left(n^{2} H+\frac{1}{2} n(n-1) a\right)^{2}>n^{2} S$ from $(2.24)$. Since the second equality in (2.26) holds, we have $|\nabla H|=0$ and hence $H$ is constant on $M^{n}$.

If $(n-1) a^{2}+4 n\left(c_{2}-b\right)=0$, since the equality holds in (2.24), we have $\left(n^{2} H+\frac{1}{2} n(n-1) a\right)^{2}=n^{2} S$. This together with (2.21) forces that

$$
S_{k}^{2}=4 n^{2} S H_{k}^{2}, \quad k=1, \ldots, n .
$$

Since the equality holds in (2.22), there exists a real function $c_{k}$ on $M^{n}$ such that

$$
h_{i j k}=c_{k} h_{i j}, \quad i, j=1, \ldots, n,
$$

for every $k$. Taking the sum on both sides of equation (2.28) with respect to $i=j$, we get

$$
H_{k}=c_{k} H, \quad k=1, \ldots, n .
$$

From (2.28), we have

$$
S_{k}=2 \sum_{i, j} h_{i j} h_{i j k}=2 c_{k} S, \quad k=1, \ldots, n .
$$

Multiplying both sides of equations in (2.30) by $H$ and by using (2.29), we have

$$
H S_{k}=2 H_{k} S, \quad k=1, \ldots, n .
$$

It follows from (2.27) and (2.31) that

$$
H_{k}^{2} S=H_{k}^{2} n^{2} H^{2}, \quad k=1, \ldots, n .
$$

Hence we have

$$
|\nabla H|^{2}\left(S-n^{2} H^{2}\right)=0 .
$$

Suppose that $H$ is not constant on $M^{n}$, we assert that $S=n^{2} H^{2}$. In fact, since $H$ is not constant, we have that $|\nabla H|$ is not vanishing identically on $M^{n}$. We denote $M_{0}=\{x \in M|| \nabla H \mid \neq 0\}$, then $M_{0}$ is open in $M$. Let $T=S-n^{2} H^{2}$, it follows form (2.33) that $T=0$ in $M_{0}$. From the continuity of $T$, we have that $T=0$ on the closure $\operatorname{cl}\left(M_{0}\right)$ of $M_{0}$. If $M / \operatorname{cl}\left(\mathrm{M}_{0}\right) \neq \emptyset$, then $H$ is constant in $M / \mathrm{cl}\left(\mathrm{M}_{0}\right)$. It follows from (2.20) that $S$ is constant and hence $T$ is constant in $M / \mathrm{cl}\left(\mathrm{M}_{0}\right)$. From the continuity of $T$, we have that $T=0$ on $M^{n}$ and hence $S=n^{2} H^{2}$. It follows from $(2.4)$ that $n(n-1) R=\sum_{i, j} \bar{K}_{j i i j}$. Since $\sum_{i, j} \bar{K}_{j i i j}$ is constant, we have that $R$ is constant and hence $H$ is constant. This is contradict to the assumption. Hence the mean curvature $H$ is constant.

Lemma 2.4 ([7]). Let $M$ be a complete Riemannian manifold whose Ricci curvature is bounded from below. Let $f$ be a $C^{2}$-function which is bounded from above. Then there exists a sequence $\left\{q_{k}\right\}$ such that

$$
\lim _{k \rightarrow \infty} f\left(q_{k}\right)=\sup f, \quad \lim _{k \rightarrow \infty}\left\|\nabla f\left(q_{k}\right)\right\|=0, \quad \limsup _{k \rightarrow \infty} L f\left(q_{k}\right) \leq 0,
$$


where $L f=\sum b_{j} f_{j j}, b_{j} \geq 0$ is bounded.

\section{Proof of theorems}

Proof of Theorem 1.4. We choose $e_{1}, \ldots, e_{n}$ such that $h_{i j}=\lambda_{i} \delta_{i j}$, then (2.13) becomes

$$
\begin{aligned}
L(n H)= & \sum_{i, j, k} h_{i j k}^{2}-n^{2}|\nabla H|^{2}-S \sum_{k} \bar{K}_{n+1 k n+1 k}-n H \sum_{i} \lambda_{i} \bar{K}_{n+1 i i n+1} \\
& -\frac{1}{2} \sum_{i, k}\left(\lambda_{i}-\lambda_{k}\right)^{2}\left(\bar{K}_{i k i k}+R_{i k i k}\right) .
\end{aligned}
$$

Next we estimate the right hand of formula (3.1) one by one. Using (1.1) and (1.2), we have

$$
\begin{aligned}
& -S \sum_{k} \bar{K}_{n+1 k n+1 k}-n H \sum_{i} \lambda_{i} \bar{K}_{n+1 i i n+1} \\
= & \sum_{k}\left(S-n H \lambda_{k}\right) \frac{c_{1}}{n}=c_{1}\left(S-n H^{2}\right),
\end{aligned}
$$

and

$$
\begin{aligned}
-\frac{1}{2} \sum_{i, k}\left(\lambda_{i}-\lambda_{k}\right)^{2}\left(\bar{K}_{i k i k}+R_{i k i k}\right) & \geq \frac{1}{2} \sum_{i, k}\left(\lambda_{i}-\lambda_{k}\right)^{2}\left(c_{2}+K_{\min }\right) \\
& =n\left(c_{2}+K_{\min }\right)\left(S-n H^{2}\right)
\end{aligned}
$$

where $K_{\text {min }}$ denotes the infimum of the sectional curvature of $M^{n}$. Substituting (3.2), (3.3) into (3.1) and from Proposition 2.3, we get

$$
L(n H) \geq \sum_{i, j, k} h_{i j k}^{2}-n^{2}|\nabla H|^{2}+n\left(K_{\min }+\frac{c_{1}}{n}+c_{2}\right)\left(S-n H^{2}\right) \geq 0,
$$

here we used the assumption $K_{\min } \geq-\frac{c_{1}}{n}-c_{2}$. Since $L$ is elliptic and $H$ can obtain its maximum on $M$, we deduce that $H$ is constant and the equalities in (3.4) hold. Thus

$$
\sum_{i, j, k} h_{i j k}^{2}=n^{2}|\nabla H|^{2}=0
$$

and

$$
\left(K_{\min }+\frac{c_{1}}{n}+c_{2}\right)\left(S-n H^{2}\right)=0 .
$$

It follows from (3.6) that either $S=n H^{2}$ and $M^{n}$ is totally umbilical or $K_{\text {min }}+\frac{c_{1}}{n}+c_{2}=0$. In the latter case, since the equality in (3.3) holds, we have that $R_{j i i j}=K_{\min }=-\frac{c_{1}}{n}-c_{2}$ and $\bar{K}_{j i i j}=c_{2}$. It follows from Gauss equation (2.2) that, for any $i, j$

$$
\lambda_{i} \lambda_{j}=\frac{c_{1}}{n}+2 c_{2} .
$$


If $\frac{c_{1}}{n}+2 c_{2}=0$, then all the $\lambda_{i}$ are zero, and $M^{n}$ is totally geodesic. Otherwise, if $\frac{c_{1}}{n}+2 c_{2} \neq 0$, we conclude that $M^{n}$ has at most two distinct principal curvature. In fact, without loss of generality, we assume that $M^{n}$ has three distinct principle curvature $\lambda_{i_{1}}, \lambda_{i_{2}}, \lambda_{i_{3}}$. Then $\lambda_{i_{1}} \lambda_{i_{2}}=\lambda_{i_{2}} \lambda_{i_{3}}=\frac{c_{1}}{n}+2 c_{2}$ and hence $\lambda_{i_{1}}=\lambda_{i_{3}}$. This is a contradiction. So $M^{n}$ has at most two distinct principal curvature. If all the principle curvatures are equal, we have that $M^{n}$ is totally umbilical. Otherwise, without loss of generality, we may suppose that

$$
\lambda_{1}=\cdots=\lambda_{k}=\lambda, \quad \lambda_{k+1}=\cdots=\lambda_{n}=\mu
$$

for some $k=1, \ldots, n-1$, and $\lambda \mu=\frac{c_{1}}{n}+2 c_{2}$. We can prove $k=1$ or $n-1$. In fact, if $1<k<n-1$, it follows from (3.7) that $\lambda^{2}=\mu^{2}=\lambda \mu=\frac{c_{1}}{n}+2 c_{2}$. This is contradict to $\lambda \neq \mu$. Hence we have $k=1$ or $n-1$.

On the other hand, it follows from (3.5) that $\lambda_{i}$ is constant for every $i$. Hence $M^{n}$ is an isometric hypersurface with two distinct principal curvatures, one of which is simple. This completes the proof of Theorem 1.4.

Proof of Theorem 1.6. When the constant $a$ vanishes, Theorem 1.3 of [21] implies that Theorem 1.6 holds. Next we assume that $a$ is not zero. It follows from Gauss formula (2.2) that (2.13) becomes

$$
\begin{aligned}
L(n H)= & \sum_{i, j, k} h_{i j k}^{2}-n^{2}|\nabla H|^{2}-S \sum_{k} \bar{K}_{n+1 k n+1 k}-n H \sum_{i} \lambda_{i} \bar{K}_{n+1 i i n+1} \\
& -\sum_{i, k}\left(\lambda_{i}-\lambda_{k}\right)^{2} \bar{K}_{i k i k}-n H \sum_{j} \lambda_{j}^{3}+S^{2} .
\end{aligned}
$$

Let $\mu_{i}=\lambda_{i}-H$ and $|\Phi|^{2}=\sum_{i} \mu_{i}^{2}$, we get

$$
\sum_{i} \mu_{i}=0, \quad|\Phi|^{2}=S-n H^{2}, \quad \sum_{i} \lambda_{i}^{3}=\sum_{i} \mu_{i}^{3}+3 H|\Phi|^{2}+n H^{3} .
$$

$M^{n}$ is totally umbilical if and only if $|\Phi|^{2}=0$. It follows from Lemma 2.1 that

$$
-n H \sum_{i} \lambda_{i}^{3} \geq-n|H| \frac{n-2}{\sqrt{n(n-1)}}|\Phi|^{3}+3 H|\Phi|^{2}+n H^{3} .
$$

From (3.2), we have

$$
-S \sum_{k} \bar{K}_{n+1 k n+1 k}-n H \sum_{i} \lambda_{i} \bar{K}_{n+1 i i n+1}=c_{1}|\Phi|^{2} .
$$

It follows from condition (1.2) that

$$
-\sum_{i, k}\left(\lambda_{i}-\lambda_{k}\right)^{2} \bar{K}_{i k i k} \geq \sum_{i, k}\left(\lambda_{i}-\lambda_{k}\right)^{2} c_{2}=n c_{2}|\Phi|^{2} .
$$

Substituting (3.9), (3.10) and (3.11) into (3.8) and from Proposition 2.3, we get

$$
L(n H) \geq|\Phi|^{2}\left(2 n c_{2}+c_{1}-n H^{2}-\frac{n(n-2)}{\sqrt{n(n-1)}}|H||\Phi|+|\Phi|^{2}\right) .
$$


Consider the quadratic form

$$
Q(x, y)=-x^{2}-\frac{n-2}{\sqrt{n-1}} x y+y^{2} .
$$

By the orthogonal transformation

$$
\left\{\begin{array}{l}
\left.u=\frac{1}{\sqrt{2 n}}\{(1+\sqrt{n-1}) y+(1-\sqrt{n-1}) x)\right\}, \\
\left.v=\frac{1}{\sqrt{2 n}}\{(1+\sqrt{n-1}) y+(1+\sqrt{n-1}) x)\right\},
\end{array}\right.
$$

the equation (3.13) becomes

$$
Q(x, y)=\frac{n}{2 \sqrt{n-1}}\left(u^{2}-v^{2}\right) .
$$

Let $x=\sqrt{n H^{2}}, y=|\Phi|$. Then $u^{2}+v^{2}=x^{2}+y^{2}=|\Phi|^{2}+n H^{2}=S$. Hence we have

$$
\begin{aligned}
2 n c_{2}+c_{1}+Q(x, y) & =2 n c_{2}+c_{1}-\frac{n}{2 \sqrt{n-1}}\left(u^{2}+v^{2}\right)+\frac{n}{\sqrt{n-1}} u^{2} \\
& \geq 2 n c_{2}+c_{1}-\frac{n}{2 \sqrt{n-1}} S .
\end{aligned}
$$

It follows from (3.12) and (3.15) that

$$
L(n H) \geq|\Phi|^{2}\left(2 n c_{2}+c_{1}-\frac{n}{2 \sqrt{(n-1)}} S\right) .
$$

Since $S \leq 2 \sqrt{n-1}\left(2 c_{2}+c_{1} / n\right)$, then $\lambda_{i}$ are bounded. It follows from $(2.20)$ that

$$
2 \sqrt{n-1}\left(2 c_{2}+c_{1} / n\right) \geq n^{2} H^{2}+n(n-1)(a H+b)-\sum_{i, j} \bar{K}_{j i i j} .
$$

Since $\bar{K}_{j i i j}$ is constant, from (3.17) we have that $H$ is bounded. Hence the Ricci curvature of $M^{n}$ is bounded from below and $n H-\lambda_{i}+\frac{n-1}{2} a$ is bounded. It follows from Lemma 2.4 that there exists a sequence $\left\{q_{k}\right\}$ such that

$$
\lim _{k \rightarrow \infty}(n H)\left(q_{k}\right)=\sup (n H), \lim _{k \rightarrow \infty}\left\|\nabla(n H)\left(q_{k}\right)\right\|=0, \limsup _{k \rightarrow \infty} L(n H)\left(q_{k}\right) \leq 0 .
$$

It follows from $(2.20)$ and $\sum_{i, j=1}^{n} \bar{K}_{i j j i}=$ constant that $\lim _{k \rightarrow \infty} S\left(q_{k}\right)=\sup S$. Evaluating (3.16) at points $q_{k}$, we have

$$
0 \geq\left(\sup S-n \sup H^{2}\right)\left(2 n c_{2}+c_{1}-\frac{n}{2 \sqrt{(n-1)}} \sup S\right) .
$$

Since $S \leq 2 \sqrt{n-1}\left(2 c_{2}+c_{1} / n\right)$, we have

$$
\sup \left(S-n H^{2}\right)\left(2 n c_{2}+c_{1}-\frac{n}{2 \sqrt{(n-1)}} \sup S\right)=0 .
$$

If $\sup \left(S-n H^{2}\right)=0$, then $S=n H^{2}$ and $M^{n}$ is totally umbilical.

If $\sup S=2 \sqrt{n-1}\left(2 c_{2}+c_{1} / n\right)$ and $\sup S$ is attained on $M^{n}$, then sup $H$ can be attained on $M^{n}$. This together with $L(n H) \geq 0$ forces that $H$ is constant. Then all the inequalities to obtain (3.16) become equalities. Since the equality 
in Lemma 2.1 holds, we have that $M^{n}$ has two distinct principal curvature. This completes the proof of Theorem 1.6.

\section{References}

[1] N. Abe, N. Koike, and S. Yamaguchi, Congruence theorems for proper semi-Riemannian hypersurfaces in a real space form, Yokohama Math. J. 35 (1987), no. 1-2, 123-136.

[2] K. Akutagawa, On spacelike hypersurfaces with constant mean curvature in the de Sitter space, Math. Z. 196 (1987), no. 1, 13-19.

[3] J. D. Baek, Q. M. Cheng, and Y. J. Suh, Complete space-like hypersurfaces in locally symmetric Lorentz spaces, J. Geom. Phys. 49 (2004), no. 2, 231-247.

[4] A. Brasil Jr, A. G. Colares, and O. Palmas, Complete hypersurfaces with constant scalar curvature in spheres, Monatsh. Math. 161 (2010), no. 4, 369-380.

[5] F. E. C. Camargo, R. M. B. Chaves, and L. A. M. Sousa Jr, Rigidity theorems for complete spacelike hypersurfaces with constant scalar curvature in de Sitter space, Differential Geom. Appl. 26 (2008), no. 6, 592-599.

[6] A. Caminha, A rigidity theorem for complete CMC hypersurfaces in Lorentz manifolds, Differential Geom. Appl. 24 (2006), no. 6, 652-659.

[7] Q. M. Cheng, Complete space-like hypersurfaces of a de Sitter space with $r=k H$, Mem. Fac. Sci. Kyushu Univ. Ser. A 44 (1990), no. 2, 67-77.

[8] Q. M. Cheng and S. Ishikawa, Spacelike hypersurfaces with constant scalar curvature, Manuscripta Math. 95 (1998), no. 4, 499-505.

[9] A. J. Goddard, Some remarks on the existence of spacelike hypersurfaces of constant mean curvature, Math. Proc. Cambridge Philos. Soc. 82 (1977), no. 3, 489-495.

[10] H. Z. Li, Global rigidity theorems of hypersurfaces, Ark. Mat. 35 (1997), 327-351.

[11] H. Z. Li, Y. J. Suh, and G. X. Wei, Linear Weingarten hypersurfaces in a unit sphere, Bull. Korean Math. Soc. 46 (2009), no. 2, 321-329.

[12] J. C. Liu and Z. Y. Sun, On spacelike hypersurfaces with constant scalar curvature in locally symmetric Lorentz spaces, J. Math. Anal. Appl. 364 (2010), no. 1, 195-203.

[13] J. C. Liu and L. Wei, A gap theorem for complete space-like hypersurface with constant scalar curvature in locally symmetric Lorentz spaces, Turkish J. Math. 34 (2010), no. 1, 105-114.

[14] S. Montiel, An integral inequality for compact spacelike hypersurfaces in de Sitter space and applications to the case of constant mean curvature, Indiana Univ. Math. J. 37 (1988), no. 4, 909-917.

[15] _ A characterization of hyperbolic cylinders in the de Sitter space, Tohoku Math. J. (2) 48 (1996), no. 1, 23-31.

[16] M. Okumura, Hypersurfaces and a pinching problem on the second fundamental tensor, Amer. J. Math. 96 (1974), 207-213.

[17] J. Ramanathan, Complete spacelike hypersurfaces of constant mean curvature in de Sitter space, Indiana Univ. Math. J. 36 (1987), no. 2, 349-359.

[18] S. C. Shu, Complete spacelike hypersurfaces in a de Sitter space, Bull. Austral. Math. Soc. 73 (2006), no. 1, 9-16.

[19] _ Space-like hypersurfaces in locally symmetric Lorentz space, Anziam J. 50 (2008), no. 1, 1-11.

[20] Y. J. Suh, Y. S. Choi, and H. Y. Yang, On space-like hypersurfaces with constant mean curvature in a Lorentz manifold, Houston J. Math. 28 (2002), no. 1, 47-70.

[21] S. C. Zhang and B. Q. Wu, Rigidity theorems for complete spacelike hypersurfaces with constant scalar curvature in locally symmetric Lorentz spaces, J. Geom. Phys. 60 (2010), no. 2, 333-340. 
College of Sciences

SHENYANG UNIVERSITY

Shenyang 110044, P. R. China

E-mail address: danyang82@yahoo.cn 